\title{
Modified OFDM Model Based on Sub-Sliding Window Technique
}

\author{
Salih Mohammed Salih \\ Electrical Engineering Department, College of Engineering, University of Anbar \\ Anbar, Iraq \\ Tel: 96-4-790-125-5958 E-mail: dr_salih_moh@yahoo.com \\ Waleed Ameen Mahmoud \\ Electrical Engineering Department, College of Engineering, University of Baghdad \\ Baghdad, Iraq \\ Tel: 96-4-790-356-8118Ｅ-mail: profwaleed54@yahoo.com
}

\begin{abstract}
In modern wireless communication systems, Doppler frequency reduction is employed for efficient transmission of data in noisy environments. Achieving a very less bit error rate (BER) has been the major task in the orthogonal frequency division multiplexing (OFDM), which is a popular multicarrier modulation technique in IEEE 802.11a wireless systems and is currently being used in the IEEE 802.16e (WiMax) standard. This paper presents a modified sub-sliding window (SSW) technique that is used to improve the performance of OFDM modem for the physical layer of IEEE 802.11a standard. To carry out the simulations, the bit error rate performance of the modified and standard models is compared under the additive white Gaussian noise (AWGN) and flat fading channel. The simulation results show that the combination of SSW and OFDM technology is superior to the OFDM standard technology.
\end{abstract}

Keywords: SSW, OFDM, Doppler Frequency, AWGN, IEEE 802.11a

\section{Introduction}

In multicarrier approach the original data stream is divided into $\mathrm{N}$ number of parallel streams. Then each stream is modulated with different carrier frequency and sent across the channel. This actually reduces inter-symbol interference as each stream travels at a different frequency. So considering the number of users, multicarrier approach will be the best choice for 4G wireless systems (Richard, 2006 and Van, 2000). Among the multi-carrier modulation schemes CDMA and OFDM are the two technologies the researchers concentrate on. CDMA is used in $3 \mathrm{G}$ wireless systems. OFDM has gained more interest in recent times and is used by many broadband transmissions like IEEE 802.11a and 802.11g (Richard, 2006). OFDM could be used both as the multiple access technology and modulation scheme in 4G. But OFDM in itself faces a lot of challenges (Richard, 2006). As specified by IEEE 802.11a, the physical layer is based on a 52-carrier OFDM modulation scheme including 48 subcarrier for data symbols and 4 pilot subcarriers for channel estimation process (Yun, 2009). In the standard model, the transmitter includes a symbol mapper, inverse fast Fourier transform (IFFT) and cyclic extension blocks. IFFT block generates subcarriers and the cyclic extension block adds the guard time interval into the OFDM symbol. The receiver consists of the guard time removal, fast Fourier transforms (FFT), symbol demapper and synchronizer blocks.

Further studies carried out in the recent years. They aimed at reducing the BER by using the channel estimation techniques. Due to the non-orthogonality between the real and imaginary parts in OFDM signals at the receiver due to the channel effect, the demodulated real valued OFDM/QAM symbol always has imaginary valued intrinsic interference from neighboring symbols (Jinfeng, 2009 and Floch, 1995). This hinders conventional channel estimation methods for OFDM from being directly applied to OFDM/QAM. Therefore, channel estimation has been considered a big problem for OFDM/QAM in dispersive channels and attracted numerous research efforts. In order to reduce the intrinsic interference to a minimum, a pilot-based channel estimation scheme has been proposed in (Javaudin, 2003) and a preamble-based channel estimation method in (Kang, 2007), where in both cases a group of adjacent symbols are carefully selected so that the intrinsic interference at the central symbol position can be greatly reduced.

In (Holtzman, 1995), an autocorrelation based scheme for maximum Doppler-frequency estimation was proposed for single-carrier systems, where the estimate is obtained using the envelope of the received signal. In (Cai, 2003), the correlation between the tail of the OFDM symbol and the guard interval was exploited to 
estimate the Doppler frequency $\left(\mathrm{f}_{\mathrm{d}}\right)$, where the effects of intersymbol interference (ISI) was not considered. In (Krasny, 2001), the estimation of $\mathrm{f}_{\mathrm{d}}$ is obtained via a maximum-likelihood (ML) based time-domain method for TDMA and CDMA systems. The application of this algorithm to OFDM systems was presented in (Yucek, 2005), where time domain channel estimations were used to obtain the maximum Doppler frequency estimation. In this model, the channel is not estimated based on pilot subcarriers, but by using preambles and inserting frequent mid-ambles. In its frequency domain approach, the ICI is ignored. Thus, an error floor is observed. In (Gao, 2009) a method for estimating the Doppler spread in mobile OFDM systems was described. The estimation was based on phase message of time domain channel estimation over several OFDM symbols.

In (Salih, 2010), a sliding window (SW) technique was proposed to enhance the system performance of the OFDM modem for the physical layer of IEEE 802.11a standard. The performance of such system was improved under the flat and the AWGN channel. The author in (Salih, 2010) used additional two blocks (FFT and SW blocks) at the transmitter side and the inverse of these blocks at the receiver side in addition to the other main blocks (i.e. IFFT at the transmitter side and FFT at the receiver side). The SW block of length 64-bits was used in (Salih, 2010) which is the same size of FFT block. In this paper, we split the block of SW into sub-sliding window (SSW) based on shifting the signal vector bit-by-bit to the right in the transmitter side and adding them vertically as shown in section 3. The transmitted vector of length $\mathrm{N}$ will be divided into 4-subsets and we apply the SSW and the IFFT on each subset. The reverse steps will be applied at the receiver to recover the transmitted signal. The modified method can improve the system performance without using the blocks of FFT at the transmitter and its inverse at the receiver sides respectively.

\subsection{Effect of Doppler Frequency}

Motion of an antenna produces Doppler shifts of incoming received waves. OFDM has been adopted by many wireless standards such as IEEE 802.11 and 802.16 and has been implemented in many practical systems. Knowledge of mobile speeds is critical in improving the performance of multi-cell wireless communication systems. The mobile speed information is also very critical for implementing a number of physical and network layer functions such as adaptive and fast link adaptation, and accurate channel prediction. Thus, the scheduler gain due to multiuser diversity and spectral efficiency of the system can be increased (Yang, 2006). The complex baseband representation (Steele, 1992) of a wireless channel impulse response can be described by:

$h(t, \tau)=\sum_{\ell} \gamma_{\ell}(t) \delta\left(\tau-\tau_{\ell}\right)$

where $\tau_{\ell}$ is the delay of the $\ell$-th path and $\gamma_{\ell}(t)$ is the corresponding complex amplitude and $\delta$ is the delta function. Due to the motion of the vehicle, $\gamma_{\ell}(t)$ 's are wide-sense stationary (WSS) narrowband complex Gaussian processes, which are independent for different paths. The Doppler frequency $\left(f_{d}=v \cdot f_{c} / c\right)$, is related to the vehicle speed $v$ and the carrier frequency $f_{c}$, where $\mathrm{c}$ is the speed of light.

\subsection{OFDM System}

The time domain samples of an OFDM symbol can be obtained from data symbols as:

$$
X_{i, n}=\operatorname{IFFT}\left\{x_{i, k}\right\}=\sum_{k=0}^{N-1} x_{i, k} e^{j 2 \pi m k / N}, n=0, \ldots, N-1
$$

where $x_{i, k}$ is data symbol of the k-th subcarrier of the $\mathrm{i}$-th OFDM symbol and $\mathrm{N}$ is the number of subcarriers. After the guard interval addition, the samples $X_{i, n}$ are transmitted over the linear time dependent channel described in (1), with additive white Gaussian noise (AWGN) $Z_{i, n}$, with zero mean and variance of $\sigma_{z}^{2}$. In this paper, we assume the channel to be constant over an OFDM symbol, but time-varying across OFDM symbols is a reasonable assumption for low mobility. At the receiver, assuming perfect synchronization, the received samples can be expressed as:

$$
y_{i, n}=X_{i, n} * h(t, \tau)+Z_{i, n}
$$

where * stands for convolution.

After removing the guard interval, the receiver demultiplexes the received samples by using the FFT as:

$$
Y_{i, k}=F F T\left\{y_{i, n}\right\}=\frac{1}{N} \sum_{n=0}^{N-1} y_{i, n} e^{-j 2 m k / N}, k=0, \ldots, N-1
$$

and the demodulated signal $\mathrm{Y}_{\mathrm{i}, \mathrm{k}}$ can be expressed as:

$$
Y_{i, k}=x_{i, k} \cdot H(t, f)+Z_{i, k}
$$




\section{Modified Model Analysis}

The block diagram of the modified OFDM model is shown in figure 1. Note that the standard window size of 64-bits for IEEE 802.11a is divided into 4 equally parts of length 16-bits after the QAM modulation. A sliding window technique will be applied on each part (Salih, 2010). A sliding window in (Salih, 2010) is used for reducing the effect of Doppler frequency; the author applied the SW on the frame length of 64-bits in addition to the other FFT and IFFT blocks added at the transmitter and the receiver sides respectively. In this work, we won't use the FFT at the transmitter and its inverse at the receiver side, in counterwise we just use the main blocks of IFFT at the transmitter and FFT at the receiver side. The additional blocks in (Salih, 2010) used to increase the orthogonality of subcarriers and reduce the round off error come from the block of SW. To reduce the round of error, the sliding window is divided into sub-sliding window of length 16-bits each. The sliding window technique is used for enhancing the system performance under the Doppler frequency effect. Note that non-coherent combining of four short correlation periods instead of a single long block correlation is due to the potential high round off error which may occur at any bit that spreads to the other adjacent bits due to the mixing property of the suggested algorithm. Also the frequency offset results in phase rotation or shifts to the sampled signals causing reduced correlation performance between the received signal and the transmitted waveform. The longer correlation period leads to larger phase shift and poorer correlation performance. A shorter correlation period thus helps mitigate the frequency offset effect and the round off error. The output data vector after the QAM modulator is:

$[x(k)]=\left[\begin{array}{lllll}x_{1} & x_{2} & x_{3} & \ldots & \ldots \\ x_{48}\end{array}\right]$

The sliding window technique can be applied on this vector by shifting the vector bit-by-bit from the left to the right which forms a matrix, and then we add the data vertically, while neglecting the last value in each shift without circulating. The other training bits (4-bits) and the bits from 53 to 64 which are used for synchronization or zero padding (In this paper, we set them to zero) are added to eq. (6) such that the total vector will be of length 64 bit. The shifting of bits will be divided into four parts according to the following step:

Shift 1: from bit 1 to 16

$$
x(n 1)=\sum_{k=1}^{n 1} x(k) \quad n_{1}=1, \ldots, N / 4
$$

Shift 2: From bit 17 to 32

$$
x\left(n_{2}\right)=\sum_{k=N / 4+1}^{n_{2}} x(k) \quad n_{2}=N / 4+1, \ldots, N / 2
$$

Shift 3: from bit 33 to 48

$$
x\left(n_{3}\right)=\sum_{k=N / 2+1}^{n_{3}} x(k) \quad n_{3}=N / 2+1, \ldots, N-N / 4
$$

Shift 4: from bit 49 to 64 , the details of these equations (i.e. eqs (7) to (10)) are given in appendix-A

$$
x\left(n_{4}\right)=\sum_{k=N-N / 4+1}^{n 4} x(k) \quad n n_{4}=N-N / 4+1, \ldots, N
$$

Now the output vector from equations (7) to (10) can be converted from frequency to time domain by using the IFFT block as in the conventional model (eq. (2)). At the receiver side, the inverse steps will be applied in order to recover the transmitted signal, where the last step in the transmitter side is the first step at the receiver side and so on. The reversing of sub-sliding window can be executed as in the following steps after the FFT block (eq. (4)):

- Consider the output data from the FFT block are:

$$
F F T_{o / p}=\bar{y}_{k}, \quad 1 \leq k \leq N
$$

- Then the reversing of sliding window can be applied on this data vector as given in the next equation $y_{1}=\bar{y}_{1}, \quad y_{2}=\bar{y}_{2}-\bar{y}_{1}, \quad y_{3}=\bar{y}_{3}-\bar{y}_{2}, \quad . \quad . \quad . \quad, y_{64}=\bar{y}_{64}-\bar{y}_{63}$ or in general form: $y_{N}=\bar{y}_{N}-\bar{y}_{N-1}$

Note that the last equation can be applied from bit 1 to N; because dividing of FFFs into four sub-blocks will not cause any effect on the inverse of sub-sliding window (ISSW). The training sequence will be used to estimate the channel frequency response at the receiver side. The channel frequency response can be applied as shown in figure 1. (Salih, 2010 and Salih, 2007)

$$
\text { Channel_Estimation }(i)=\frac{\operatorname{Re} \text { ceived Training }(i)}{\text { Transmitted Training }(i)}, \quad 1 \leq i \leq 4
$$


The length of 4-bits of training vector can be increased by repeating them where the total length will be the same as the total number of active subcarriers which are 48-bits. The channel frequency response which is found in the last step will be used to compensate the channel effect on the data, and the estimated data can be found using the following equation:

Estimated Data $(j)=(\text { Channel Estimation }(j))^{-1} \times \operatorname{Re}$ ceived Data $(j), \quad$ where $j=1$ to 48

The final step is to apply the QAM demodulation at the recovered data after channel estimation and compensation.

\section{Effectiveness of Sliding Window Technique}

The effect of Doppler spread in wireless channels in OFDM systems is important, since channel variations during one OFDM symbol cause Inter Sub-Carrier Interference (ICI), which degrades its performance. As the fluctuation of transmitted signal shape reduces, the Doppler frequency has less effect on the system performance (Salih, 2010). To show the effect of our suggested SW technique on the BER performance of OFDM model, let us consider that a sine wave signal is discretized into 32 bit and transmitted over wireless channel. This signal is subjected to a Doppler frequency of 1.1 of the carrier frequency with respect to the main transmitted signal frequency as shown in figure 2. The transmitted signal shape has blue solid line curve (TX without SW), while the received signal has black line curve (RX without SW). From this curve it is clear that there is a phase shift between them due to Doppler frequency effect. If we set the threshold at ' 0 ' level, then the received signal is considered as logic ' 1 ' if it is more than this level, else it will be of logic ' 0 ' value. From this figure, there is an error of 7-bits due to Doppler Effect on the received signal.

If the SW technique is applied on the transmitted signal then its shape will change (black line with a circle) (Note 1), the other line (black with a square); represents the received signal after adding the Doppler frequency. The last dotted line represents the received signal shape after applying the inverse of sliding window (ISW). From this curve (dotted Line), it can be seen that the signal shape, after applying the SW technique, is nearer to the shape of the main transmitted signal (TX without SW), and the error is about 0.42 of the total error that appears in RX without SW (only 3-bits of error relative to the 7-bits without SW), so the proposed algorithm reduces the effect of Doppler frequency.

\section{Simulation Results}

The Performance of the proposed and standard models can be tested under the flat fading and AWGN channel. The standard parameters are given in table (1) (Richard, 2006, Salih, 2010, and Gavin, 2004)

\subsection{The Performance at the AWGN Channel}

The performance of modified and standard models at the AWGN channel and at two values of constellation mapping points (16QAM, and 64QAM points. i.e. $\mathrm{m}=16$, and 64 points) is shown in figure 3 . From this figure, it can be seen that the modified model based on SSW technique has a $B E R=10^{-2}$ at $\mathrm{SNR}=34 \mathrm{~dB}$, while the conventional model has the same BER at $37 \mathrm{~dB}$, which means that we obtained a gain of $3 \mathrm{~dB}$ from the modified model. As the QAM points increases from 16 to 64 (dotted curves), the BER rate for both models will also increase. Wide span gains between the performances of these models are obtained, so that the modified model is better and more significant than the conventional system at the AWGN channel. The BER increases in the same level for both models, and this ensures that the modified model has a stable performance related to the conventional system.

\subsection{The Performance of Models in Flat Fading Channel}

In this section, the performance of models in flat fading channel can be simulated at Doppler frequencies $15 \mathrm{~Hz}$, and $250 \mathrm{~Hz}$. Figure 4 shows the performance of both models at Doppler frequency $15 \mathrm{~Hz}$. A gain of $2.5 \mathrm{~dB}$ at $\mathrm{BER}=10^{-2}$ was obtained from the modified model relative to the standard one at $\mathrm{m}=16$. As $\mathrm{m}$ increases from 16 to 64 points, the BER for both models increases. In all range of SNR, the suggested model outperforms the other one. The BER was increased when the channel model varied from AWGN to flat fading.

The performance of both models at Doppler frequency $250 \mathrm{~Hz}$ is shown in figure 5 . The increasing in the Doppler frequency leads to increase the BER for both models, but the suggested model still outperforms the other standard model in all range of SNR. The obtained gain from the modified model is about $5 \mathrm{~dB}$. As the number of constellation mapping increases from 16 to 64 , the BER of models increases in the same amount

\section{Conclusion}

By carrying out the analysis, we investigated new modified model of OFDM for the physical layer of IEEE 802.11a standard by using a sub-sliding window technique. The simulation results have shown that the new 
method can improve the system performance at the AWGN and flat fading channel. We observed that the performance of sub-sliding window model is superior to that of the conventional model, where different gains obtained from the proposed model relative to the standard model. We proposed a sub-sliding window transceiver for OFDM over AWGN and flat fading channel, and analyzed its performance. We also compared the performance of models by using two values of the constellation of the modulation to be optimized for SNR; we found that the modified OFDM have an advantage over standard OFDM in terms of the simulated BER performance.

\section{References}

Richard Vun Nee. (2006). A New OFDM standard for high rate wireless LAN in the 5 GHZ band, IWCMC'06, July 3-6, Vancouver, British Columbia, Canada.

Richard van Nee, Prasad R. (2000). OFDM Wireless Multimedia Communications. Artech House, Boston.

Yun LI, Jie Zhou, Yoshikuni Onozato. (2009). Performance Study for STC-OFDM Systems Based on IEEE802.11a Standard, $5^{\text {th }}$ International Conference on Wireless communications, Networking and Mobile Computing, WiCom 09, pp. 1-4.

Jinfeng Dut, Svante Signell. (2009). Novel Preamble-Based Channel Estimation for OFDM/OQAM Systems, proceeding at IEEE ICC 2009.

Floch B Le., Alard M. and Berrou C. (1995). Coded Orthogonal Frequency Division Multiplex, Proceedings of the IEEE, pp. 982-996.

Javaudin J. P., Lacroix D. and Rouxel A. (2003). Pilot-aided channel estimation for OFDM/OQAM, VTC'03 Spring, pp. 1581-1585.

Kang S.W., Chang K. H. (2007). A novel channel estimation scheme for OFDM/OQAM-IOTA system, ETRI Journal, vol. 29, pp. 430-436.

Holtzman J. M., Sampath A. (1995). Adaptive averaging methodology for handoffs in cellular systems, IEEE Trans. on Vehicular Technology1995, vol. 44, no. 1, pp. 59-66.

Cai J., Song W., and Li Z. (2003). Doppler spread estimation for mobile OFDM systems in Rayleigh fading channels, IEEE Trans. on Consumer Electronics, vol. 49, pp. 973-977.

Krasny L., Arslan H., Koilpillai D., and Chennakeshu S. (2001). Doppler spread estimation in mobile radio systems, IEEE Communication Letters, vol. 5, no. 5, pp. 197-199.

Yucek T., Tannious R. M. A., and Arslan H. (2005). Doppler spread estimation for wireless OFDM systems, IEEE/Sarnoff Symposium on Advances in Wired and Wireless Communication, pp. 233-236.

Gao Yuan, Chen Wei, Xie Tao, Long Biqi. (2009). Doppler Spread Estimation for Non-Rayleigh Fading Channel, IEEE conference, proceeding at ICIEA 2009, pp. 1106-1109.

Salih Mohammed S. (2010). Novel Sliding Window Technique of OFDM Modem for the Physical Layer of IEEE 802.11a Standard, Journal of Communications, Vol, 3. Issue 1, pp. 67-71.

Yang-Seok Choi, Can Ozdural O., Huaping Liu, Siavash Alamouti. (2006). A Maximum Likelihood Doppler Frequency Estimator for OFDM Systems, Proceedings at IEEE ICC 2006, pp.4572-4576.

Steele R. (1992). Mobile Radio Communications. New York: IEEE Press.

Gavin Yeung, Mineo Takai, Rajive Bagrodia, Alireza Mehrnia, Babak Daneshrad. (2004). Detailed OFDM Modeling in Network Simulation of Mobile Ad Hoc Networks, Proc. of the $18^{\text {th }}$ Workshop on Parallel and Distributed Simulation (PADS'04), pp. 26-34.

Salih Mohammed, Ahmed A., Adnan S., Ahmed N. (2007). Improvement model of MC-CDMA in frequency selective fading channel, the second international conference on cognitive radio oriented wireless network and communication, (CROWNCOM 2007), Orlando-Florida, USA.

Note 1: The real transmitted signal shape has random amplitude and phase depending on the input discrete data, but we assumed it has sinewave shape such that the reader can simulate fig. 2. easily for understanding the idea. If we take the output from the IFFT for a random input signal, then all curves in fig. 2 . will change. 
Table 1. Simulation Parameters

\begin{tabular}{|c|c|}
\hline Modulation Type=QAM & 16QAM, 64QAM \\
\hline Guard Period & 8-bits \\
\hline Doppler spread & $15,250 \mathrm{~Hz}$ \\
\hline Pilot subcarriers & 4 \\
\hline Number of effective sub-carriers & 48 \\
\hline Number of transmitted symbols & 24000 \\
\hline Channel frequency & $5.2 \mathrm{GHz}$ \\
\hline Data rate & $54 \mathrm{Mbps}$ \\
\hline \multirow{2}{*}{ Channel model } & AWGN \\
\cline { 2 - 2 } & Flat fading+AWGN \\
\hline
\end{tabular}

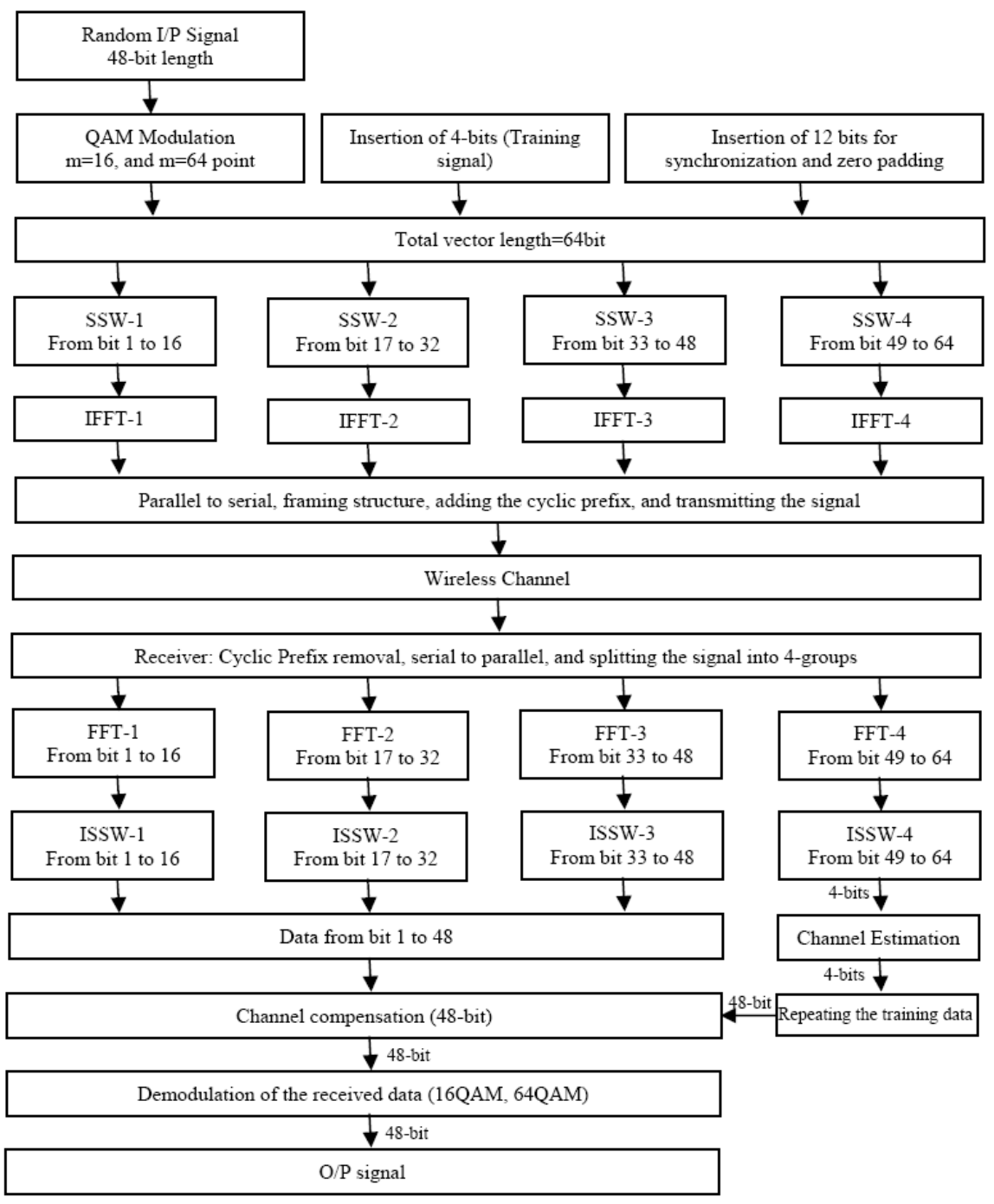

Figure 1. Block diagram of the proposed model 


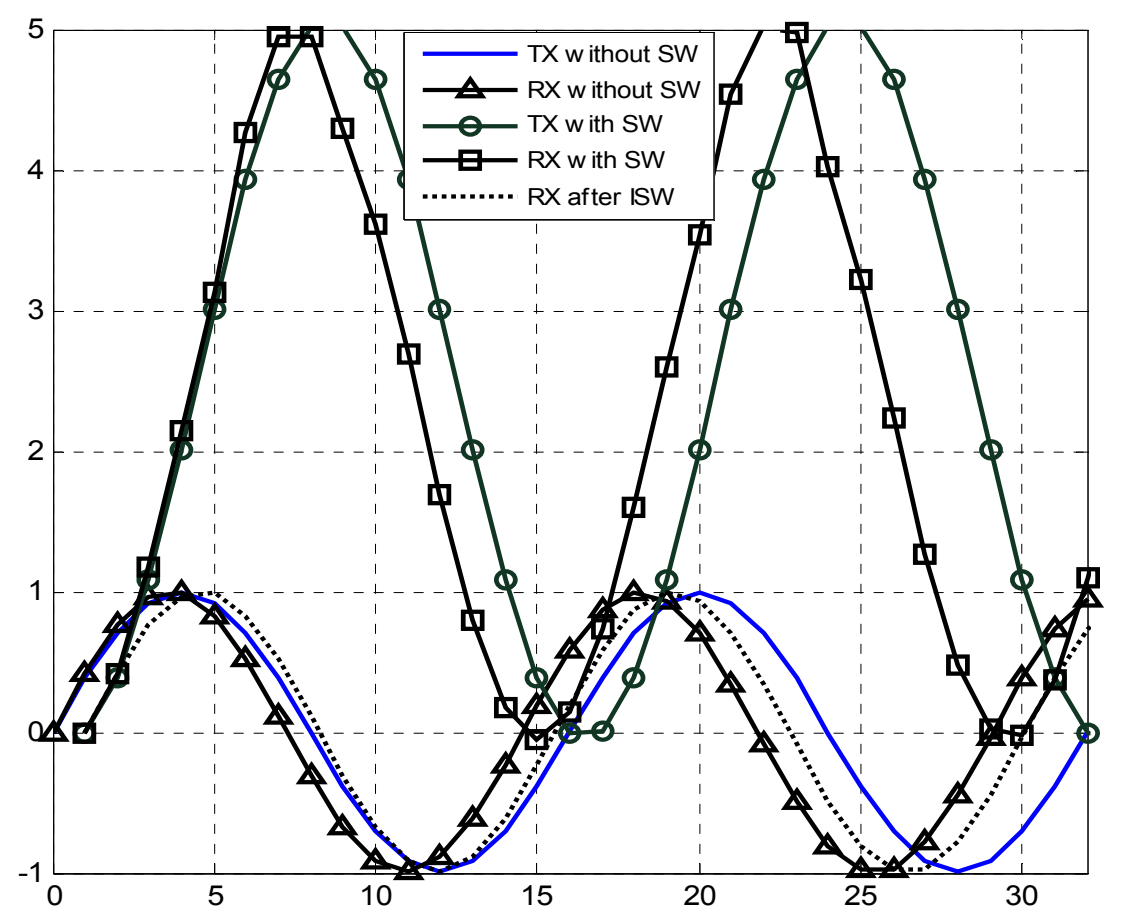

Figure 2. Doppler Effect on the system performance

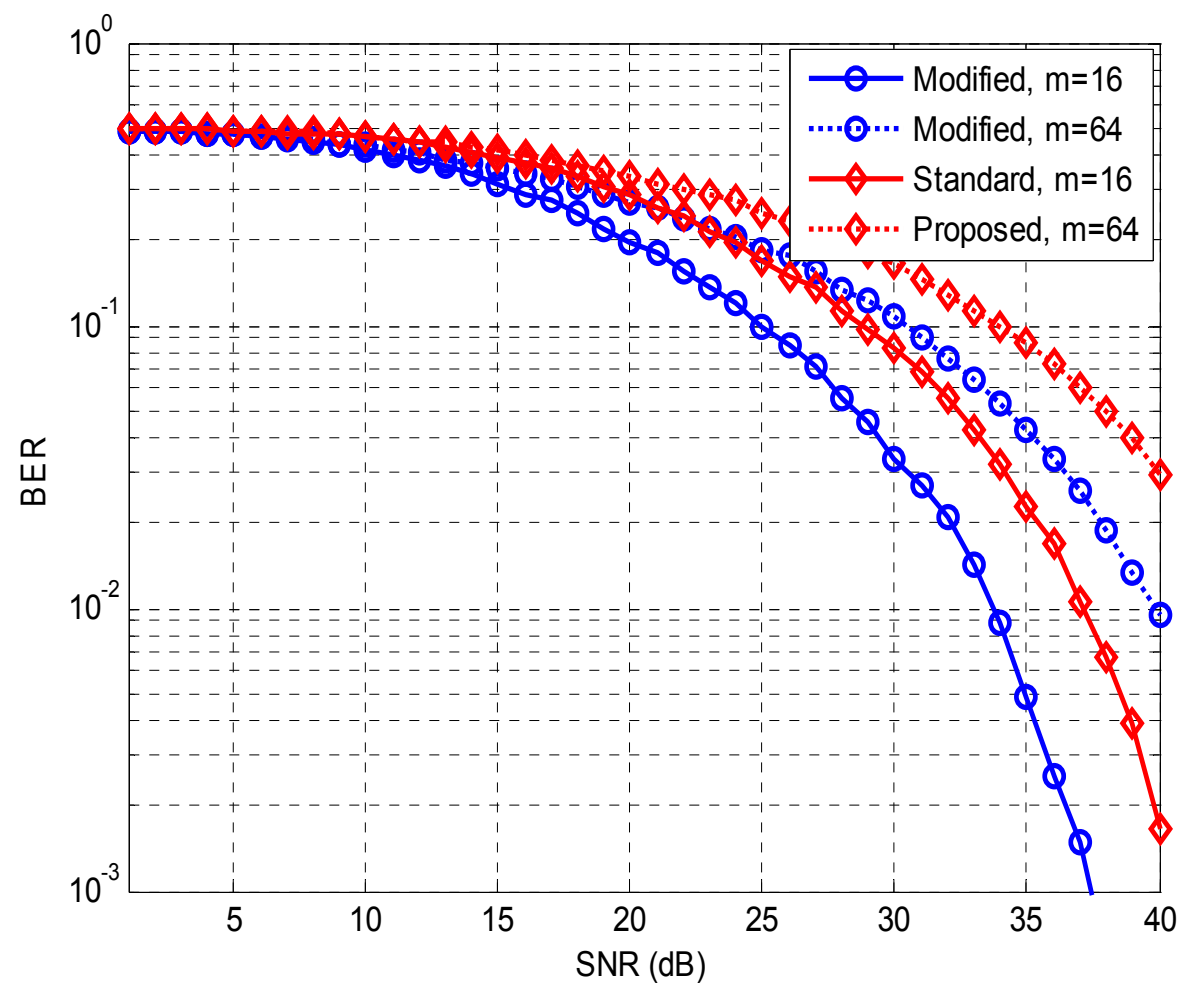

Figure 3. Performance of standard and modified model at the AWGN channel 


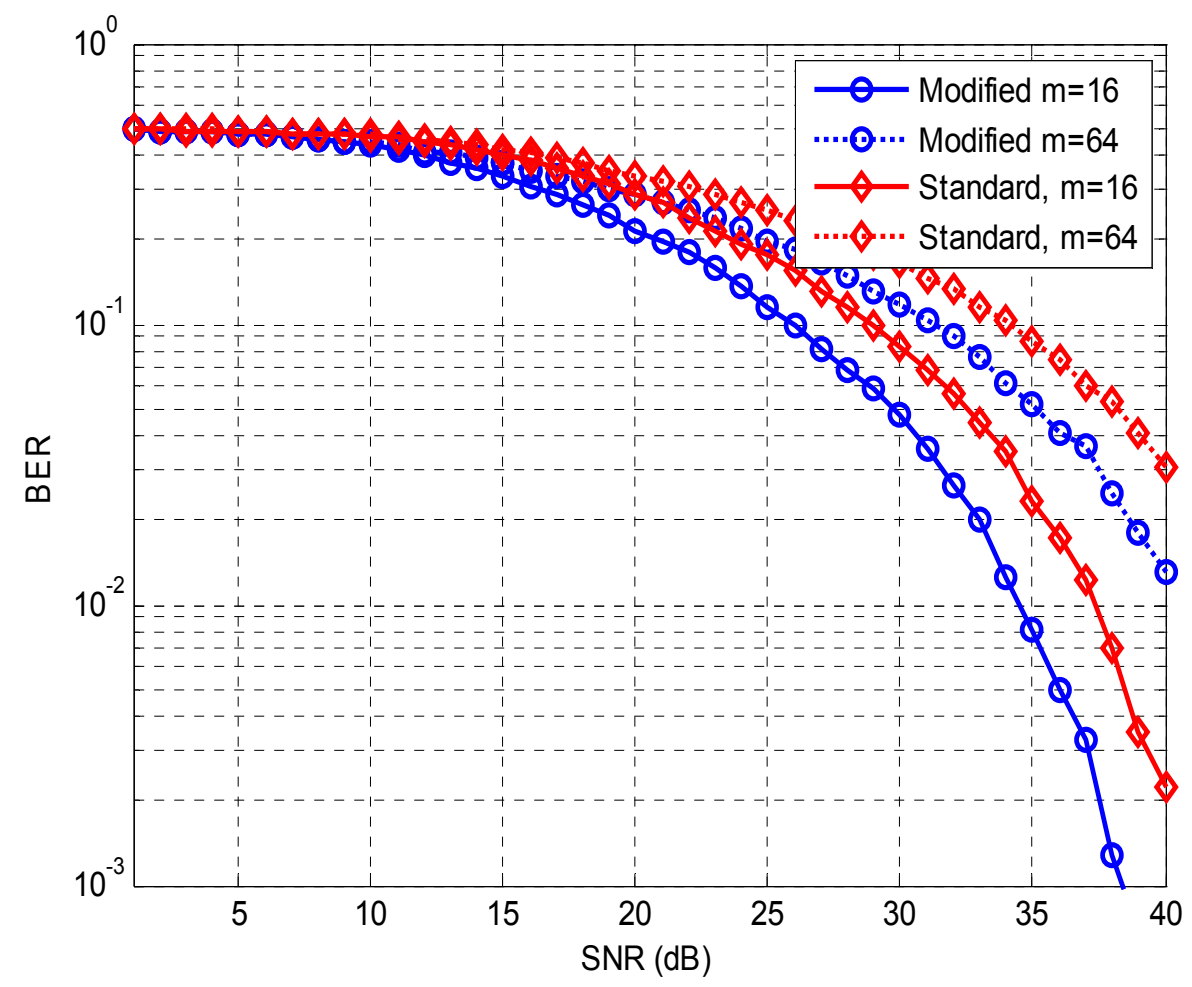

Figure 4. Performance of standard and modified models in flat fading channel (Doppler frequency $=15 \mathrm{~Hz}$ )

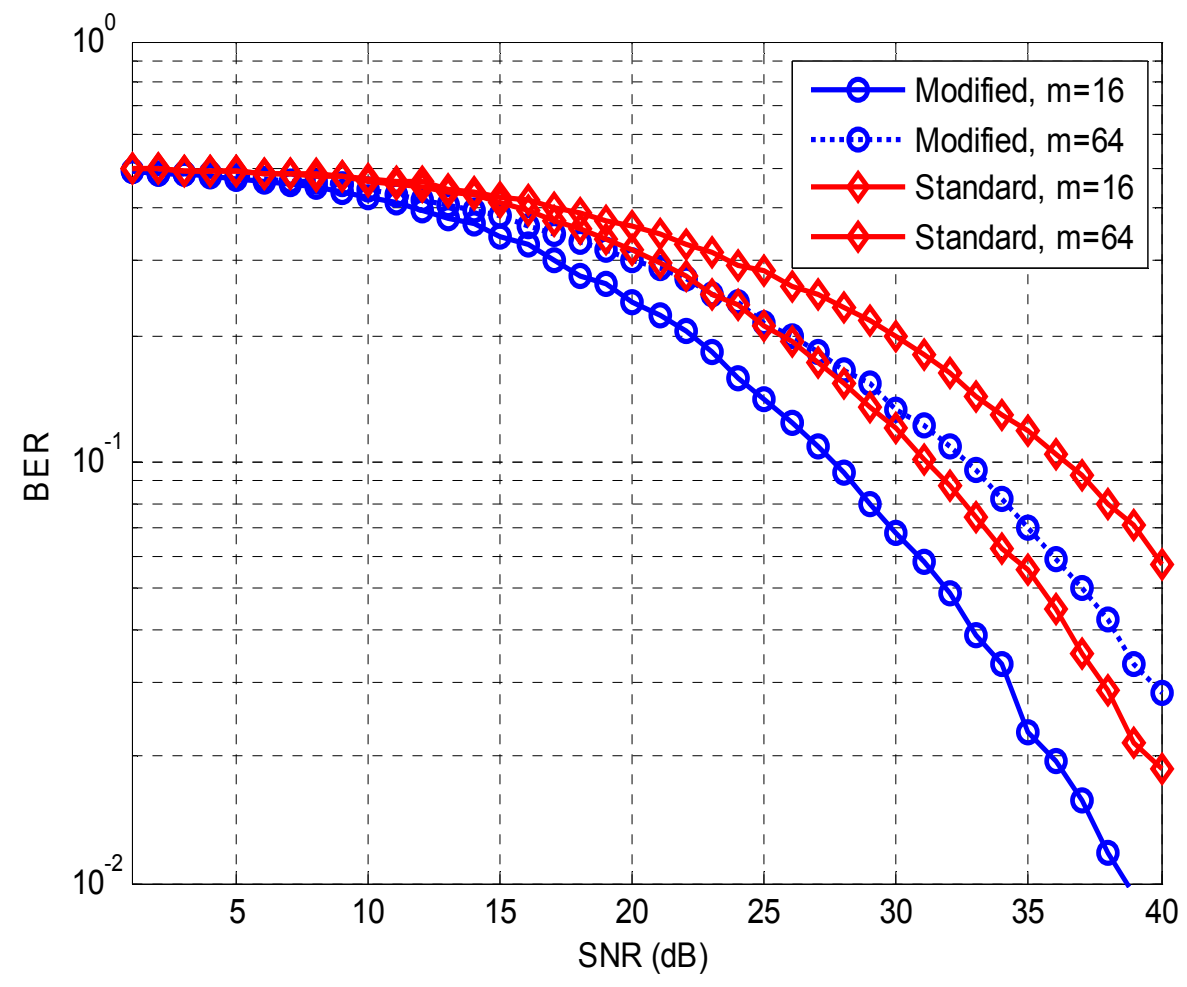

Figure 5. Performance of standard and modified models in flat fading channel (Doppler frequency $=250 \mathrm{~Hz}$ ) 


\section{Appendix A}

The sliding window technique from equations (7) to (10) can be applied on (6) by shifting the vector bit-by-bit from the left to the right as in the next equation, while neglecting the last value in each shift without circulating. Also the shifting of bits will be divided into four parts according to the following step:

Shift 1: from bit 1 to 16

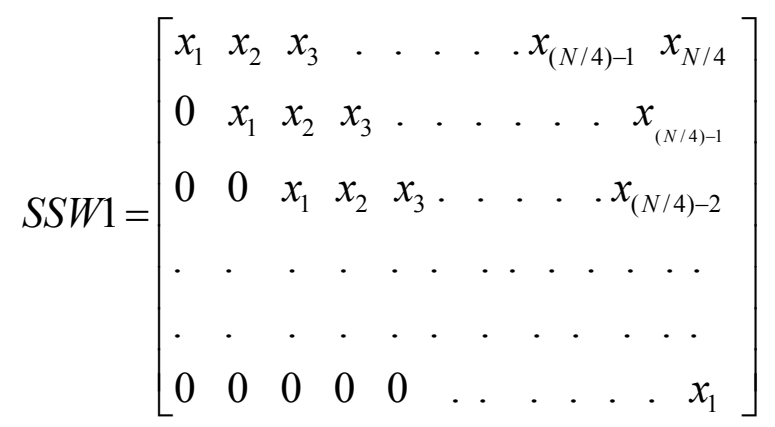

Shift 2: From bit 17 to 32

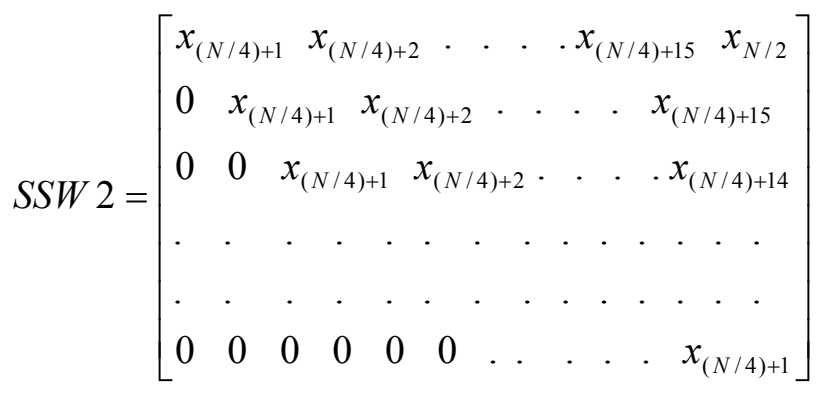

Shift 3: from bit 33 to 48

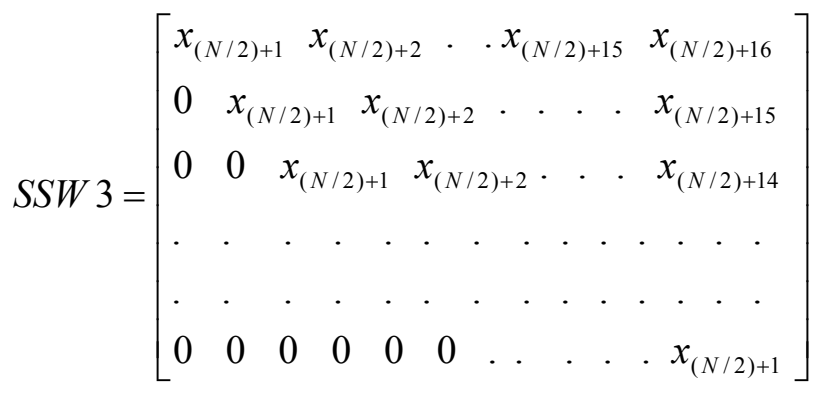

Shift 4: from bit 49 to 64 , where the bits from 53 to 64 used for synchronization or zero padding. In this paper, we set them to zero.

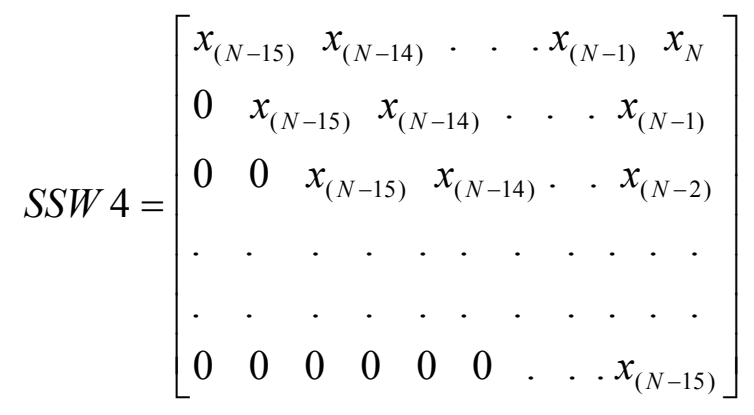

After applying these formulas of SSW by shifting the data vectors horizontally, we add the values vertically as given by the next four equations. The output from these equations will be of the same length of the input data vector. (i.e. $\mathrm{N}=64$ bits). 
The output data from bit 1 to 16 are:

$$
\begin{aligned}
& \bar{x}_{1}=x_{1} \\
& \bar{x}_{2}=x_{2}+x_{1} \\
& \bar{x}_{3}=x_{3}+x_{2}+x_{1} \\
& \cdot \quad \cdot \quad \cdot \quad \cdot \quad \cdot \\
& \cdot \quad \cdot \quad \cdot \quad \cdot \quad \cdot \quad \cdot \\
& \bar{x}_{(N / 4)}=x_{(n / 4)}+x_{(n / 4-1)}+x_{(n-2)}+. \quad \cdot \quad \cdot \quad+x_{3}+x_{2}+x_{1}
\end{aligned}
$$

From bit 17 to 32 are:

$$
\begin{aligned}
& \bar{x}_{(n / 4+1)}=x_{(n / 4+1)} \\
& \bar{x}_{(n / 4+2)}=x_{(n / 4+2)}+x_{(n / 4+1)} \\
& \bar{x}_{(n / 4+3)}=x_{(n / 4+3)}+x_{(n / 4+2)}+x_{(n / 4+1)} \\
& \text {. . . . } \\
& \bar{x}_{(N / 2)}=x_{(n / 2)}+x_{(n / 2-1)}+x_{(n / 2-2)}+. \quad . \quad .+x_{(n / 2-14)}+x_{(n / 2-15)}
\end{aligned}
$$

from bit 33 to 48 are:

$$
\begin{aligned}
& \bar{x}_{(n / 2+1)}=x_{(n / 2+1)} \\
& \bar{x}_{(n / 2+2)}=x_{(n / 2+2)}+x_{(n / 2+1)} \\
& \bar{x}_{(n / 2+3)}=x_{(n / 2+3)}+x_{(n / 2+2)}+x_{(n / 2+1)} \\
& \cdot \quad \cdot \quad \cdot \quad \cdot \quad \cdot \\
& \cdot \quad \cdot \quad \cdot \quad \cdot \quad \cdot \\
& \bar{x}_{(n / 2+16)}=x_{(n / 2+16)}+x_{(n / 2+15)}+x_{(n / 2+14)}+\cdot \cdot \cdot \cdot+x_{(n / 2+2)}+x_{(n / 2+1)}
\end{aligned}
$$

and from bit 49 to 64 are:

$$
\begin{aligned}
& \bar{x}_{(n-15)}=x_{(n-15)} \\
& \bar{x}_{(n-14)}=x_{(n-14)}+x_{(n-15)} \\
& \bar{x}_{(n-13)}=x_{(n-13)}+x_{(n-14)}+x_{(n-15)} \\
& \cdot \quad \cdot \quad \cdot \quad \cdot \quad \cdot \\
& \cdot \quad \cdot \quad \cdot \quad \cdot \quad \cdot \quad \cdot+x_{(n-14)}+x_{(n-15)}
\end{aligned}
$$

Note that equations (15) and (19) represent the details of (7), equations (16) and (20) represent the details of (8), equations (17) and (21) represent the details of (9), and equations (18) and (22) represent the details of (10). 